\title{
Mitochondria and Neurodegeneration "Could Mitochondrial Organelle Transfer be a Cellular Biotherapy for Neurodegenerative Diseases?"
}

\author{
RL Elliott*, XP Jiang and JF Head
}

Elliott-Baucom-Head Breast Cancer Research and Treatment Center, Baton Rouge, LA 70806, USA

Received: 09 February, 2016; Accepted: 26 February, 2016; Published: 07 March, 2016

*Corresponding author: R.L. Elliott, Elliott-Baucom-Head Breast Cancer Research and Treatment Center, Baton Rouge, LA 70806, USA, Email: relliott@eehbreastca.com

\begin{abstract}
It has been known for some time the abnormal function of mitochondria is associated with neurodegenerative diseases. Mitochondrial dysfunction has been implicated in the pathogenesis of Parkinson', Alzheimer's, amyotrophic lateral sclerosis, and Huntington's diseases. Researchers have postulated the therapeutic efficacy of mitochondrially targeted antioxidants, and some have shown encouraging results. We have demonstrated that mitochondrial organelle transplantation of isolated normal mitochondria into cancer cells decreased proliferation, lactate production and increased drug sensitivity of the cancer cells. Studies have shown that cellular uptake of exogenous mitochondria has restored functional recovery of defective recipient cells. Based on our experience with Mitochondrial Organelle Transfer (MOT) in cancer, we present this review commentary evidence that (MOT) might be a cell-based therapy for neurodegenerative diseases.
\end{abstract}

Keywords: Mitochondrial Organelle Transfer; Neurodegenerative Disease; Biotherapy

\section{Introduction}

Mitochondria are vital intracellular organelles involved in every important essential cellular function. They are involved in the synthesis of ATP for energy production, cell signaling, and production of reactive oxygen species, iron, and cellular metabolism, autophagy, and apoptosis. Paradoxically, they are involved in cell survival and cell death. Neurodegenerative diseases have long been associated with mitochondrial dysfunction, and many researchers are attempting to attack mitochondria as a therapeutic target for neurodegeneration.

In the 1930s, Warburg reported that cancer cells had altered cell metabolism due to defective mitochondrial respiration. He noticed a shift from oxidative phosphorylation to glycolysis with a marked increase in lactate production. There was a production of lactate in the presence of oxygen without an increase in oxidative phosphorylation. Thus, this aerobic glycolysis became known as the Warburg effect [1,2]. Our work on MOT has so far only involved cancer and has been encouraging. We have demonstrated that isolated normal mitochondria could easily enter into cancer cells when cocultured. The uptake of normal mitochondria into the cancer cells inhibited proliferation, increased drug sensitivity and decreased lactate production [3]. These results suggested that if MOT might be a cell-based therapy for cancer, it could also be a possible therapy for neurodegenerative diseases and other mitochondrial disorders. Mitochondria are involved in the longevity of the organism, and mitochondrial diseases are debilitating and occasionally fatal.

\section{Disorders and Diseases Associated With Mitochondrial Dysfunction}

Mitochondrial dysfunction is associated with many conditions besides cancer. Some of these conditions are aging, diabetes mellitus, Friedreich's Ataxia, Parkinson's disease, Amyotrophic Lateral Sclerosis (ALS), Alzheimer's and Huntington's diseases. This fact documents the important role mitochondria play in health and disease. This is discussed in detail in a very complex paper by Nunnari and Suomalainen entitled "Mitochondria in sickness and in health" [4]. This is a great article about all aspects of mitochondrial function in health and disease, and it is highly recommended. However, it is not for the novice. Now we know the result of MOT in cancer, it is appropriate to begin research to gain evidence that MOT could possible impact the treatment of these other diseases. If MOT could palliate and improve the condition of patients with ALS, it would be a tremendous hope for these fatal disease patients.

\section{Intercellular Mitochondrial Migration: Evidence they Might be an Organelle for Cellular Biotherapy}

Mitochondria are dynamic intracellular organelles that are active undergoing constant fission and fusion. They are intimately connected and networked with other cellular organelles. Their functions extend beyond cell membranes and controls organisms physiology by communications between cells, tissues and organs.

Parquier et al. [5] reported on the preferential transfer of mitochondria from endothelial cells to cancer cells through tunneling nanotubes. This transfer mediates cytoplasmic transfer and phenotype exchange between cancer and 
endothelial cells. This mitochondrial transfer resulted in an acquired chemoresistance. Another interesting paper supporting MOT as a possible treatment for neurodegenerative diseases was recently published by Kitani et al. [6]. The paper is entitled "Direct human mitochondrial transfer: a novel concept based on the endosymbiotic theory". They demonstrated isolated human mitochondria will internalize into isogeneic mesenchymal cells. They cited our paper [3] and their research support that transfer of exogenous mitochondria into diseased human cells could be a mechanism of cell-based therapy. This concept inspires new areas of research on tumorigenesis and allows for the development of new therapies for neurodegenerative disease and cancer.

\section{Neuro Degeneration: Evidence of Mitochondrial Dysfunction}

There is a strong evidence that mitochondrial dysfunction plays a significant role in neurodegenerative processes and diseases. We will attempt to present some of this strong evidence, and how MOT might play a role in therapy. Menzies, Cookson and Taylor et al. [7] have discussed in detail mitochondrial dysfunction in a cell culture model of Familial Amyotrophic Lateral Sclerosis (FALS). Evidence indicates mitochondrial abnormalities probably develop during motor neuron injury. They developed a cell culture model of FALS. The motor Neuron Cell Line (NSC-34) has been stably transfected to express normal or mutant Superoxide Dismutase (SOD1). They showed that presence of mutant (SOD1) results in the development of abnormally swollen and pale staining mitochondria. These morphological changes were associated with biochemical problems with a specific decrease in the function of complex II and IV of the mitochondrial Electron Transport Chain (ETC). Therefore, therapeutic measures protect mitochondrial respiration and may be helpful in SOD1 related familial and other forms of ALS.

Emerging evidence suggested motor neurons may die a programmed cell death pathway [8], and in relation to these factors, there are specific cell features which could cause motor neurons to be susceptible to injury [9]. There has been considerable interest in the possibility that mitochondrial damage may contribute to age-related motor neuronal injury $[10,11]$. Mitochondrial pathology of neurodegenerative disease includes generation of intracellular ATP, buffering of intracellular calcium, generation of intracellular free radical species and involvement of programmed cell death (Apoptosis). Mitochondrial proteins and DNA are shown to be susceptible to oxidative stress and free radicals that inhibit the function of specific mitochondrial enzymes [12]. Kong and Xu reported some early features of motor neuron injury occurring in transgenic mouse models of SOD1 related ALS. Before the animals developed clinical signs of motor dysfunction there was an appearance of morphologically abnormal mitochondria which were swollen and vacuolated [13]. All of this data clearly suggested that mitochondria may represent a subcellular organelle susceptible to damage in ALS. These mitochondrial alterations demonstrated in the cell culture model of SOD1 related FALS suggest that therapeutic measures targeted to the protection of mitochondrial respiratory chain function could be useful in that subgroup of ALS patients [7]. Why

\section{not attempt normal isolated mitochondrial transfer?}

A recent paper explains that how healthy mitochondria maintain proper neuronal function. This required a balance of biogenesis of new mitochondria and removal of damaged mitochondrial membranes, proteins and DNA (Mitophagy) $[14,15]$. Recent evidence suggests mitochondria are actively transported from the cell body and the axon, but a paper by Davis et al. [16] offers an interesting alternative. They state that axons expel mitochondria to the neighboring astrocytes for degeneration. This process represents a major route for mitochondrial disposal from these neurons. Davis et al. [16] have named this process of transcellular degeneration of mitochondria Trans mitophagy. Therefore, the health of the cell depends on critical mechanisms to remove damaged mitochondrial proteins and lipids. Mitochondrial quality control is essentially a complicated process, and there are many pathways of mitophagy.

Soubannier and Rippstein et al. [17] have reported the role of mitochondria derived vesicle formation in mitochondrial quality control and the molecular mechanisms that govern selective mitophagy. They describe a new pathway for the selective removal of proteins by Mitochondria Derived Vesicles (MDV) that are carriers of transit cargo to the lysosome. These vesicles carry a selective enrichment of oxidized cargo. They documented the ability of mitochondria to produce MDV that selectively transports their cargo to either the lysosomes or peroxisomes. Their conclusion states that the cargo into the MDV is very selective and can include one or both mitochondrial membranes and is enriched for oxidized protein. All in all MDV delivery of mitochondrial cargo to lysosomes is a significant new pathway for mitochondrial quality control especially in neurons. Any defect in this delicate mitophagy pathway could certainly contribute to mitochondrial dysfunction which might lead to neuro degeneration.

Mitochondrial physiology function is very delicate in the brain, spine, and their multiple neurons. It is easy to see how an imbalance in the mitochondrial function in certain neurons could lead to severe neuronal malfunction and various neurodegenerative diseases.

\section{Mitochondria: Oxidative Stress a Therapeutic Target in Neurodegeneration}

Federico, Cardaioli E, Da Pozzo et al. [18] have published a great paper on the role of mitochondrial oxidative stress and neurodegeneration. They did a great job explaining how the brain and central nervous system has high energy demands. This ATP energy is supplied by mitochondrial Oxidative Phosphorylation (OXPHOS). This OXPHOS requires many mitochondrial redox enzymes to prevent cellular damage from Reactive Oxygen Species (ROS). Any naturally occurring in efficiencies of mitochondrial OXPHOS can generate damaging ROS and cause defective mitochondrial respiration. Whatever the mechanism, a common feature of mitochondrial dysfunction is defective respiratory chain activity resulting in mitochondrial disorders and a wide range of clinical diseases. They also discuss in detail many of the individual mitochondrial disorders and diseases. The 
causes, mitochondrial defects and possible therapeutic measures are presented. This article is highly recommended.

George Perry has contributed tremendously to our knowledge of neurodegeneration and the role of defective mitochondria and mitochondrial dysfunction. He and his colleagues, Moreira, Zhu and Wang et al. have published a paper entitled "Mitochondria: a therapeutic target in neurodegeneration". They discuss the continuous energy requirement of the brain and neurons, and how even small periods of glucose and oxygen deprivation can result in neuronal cell death. They state that although the brain represents only $2 \%$ of body weight, it gets $15 \%$ of cardiac output and $20 \%$ of total body oxygen consumption. They mention how if oxidative stress occurs and excessive free radicals are produced, it can overwhelm the cell's antioxidant defenses to neutralize them. This is followed by mitochondrial dysfunction and neuronal damage.

The roles of mitochondrial dysfunction in neurodegeneration of the many neurodegenerative diseases are discussed in detail. Mitochondrial damage and the mechanismoftheenzymesinvolved in these diseases are also presented. Multiple antioxidants are discussed as therapies targeted to the mitochondrial dysfunction in these conditions. The mechanism of an action in each of the enzyme defect in these individual neurodegenerative disorders is explained. They discuss improvements in some of these conditions and emphasize that probably some of these antioxidants should be used for prevention of some neurodegenerative disorders before the appearance of symptoms. The use of these agents for prevention possibly could delay the onset and alleviate symptoms of some of these dreaded diseases [19]. This article is a must read for those researchers interested in the pathogenesis and therapy of neurodegenerative diseases.

\section{PGC1a: Role in Mitochondrial Metabolism and Neurodegenerative Disorders}

PGC1a is an important transcriptional coactivator that is an inducer of mitochondrial biogenesis and metabolism. It is a promoter of oxidative metabolism and also a tremendous regulator of ROS control by increasing the expression of many ROS-detoxifying enzymes, thus playing a paradoxical role in mitochondrial ROS control. Therefore, PGC1a is involved in mitochondrial biogenesis and detoxification of ROS.

PGC1a is the peroxisome proliferator-activated receptor gamma coactivator 1 alpha and is a family member of transcriptional coactivators that are major regulators of metabolism. The importance of a balance in this role can be associated with an impaired mitochondrial function of ROS balance in ageing and neurodegenerative diseases.

Austin and St Pierre have done a tremendous job discussing the role of PGC1a in neurodegenerative disorders. Their paper is entitled "PGC1a and mitochondrial metabolism-emerging concepts and relevance in ageing and neurodegenerative disorders" [20]. This is a very complicated paper on the known roles of PGC1a family of transcriptional coactivators. Many roles are presented in detail, but this has also elicited unknown roles stimulating many questions that need to be answered.
They state the role of PGC1a is a regulation of oxidative metabolism and organelle biogenesis of mitochondria and peroxisomes. They showed that PGC1a increases the expression of Nuclear Respiratory Factors (NRES) which are transcriptional factors that regulate expression of many mitochondrial genes. This increase mediates a great induction of uncoupled respiration. This work identified PGC1a as a master inducer of mitochondrial biogenesis and respiration. They show how the peroxisome is a central organelle that supports mitochondrial function during oxidative metabolism. During oxidative metabolism mitochondria and peroxisomes cooperate in the metabolism of lipids which are important fuels from oxidative metabolism. The MDV we discussed earlier is important in the cooperative process.

ROS metabolism is discussed including the production of ROS and the many ROS-detoxifying enzymes in the compartments of mitochondria, peroxisomes, and the cytoplasm. PGC1a by controlling this dedicate balance has a global effect on mitochondrial functions. It confirms that PGC1a controls mitochondrial respiration in two ways,

(1) Changing number of mitochondria in cells and

(2) Changing the respiratory capacity of individual mitochondria. Therefore, PGC1a might provide a unified control of mitochondrial metabolism.

They explain because of the importance of PGC1a in energy homeostasis, how any imbalance could implicate PGC1a in many pathological conditions especially neurodegenerative disorders. They discuss pathological conditions of ageing, Huntington's disease, Parkinson's disease and Alzheimer's Disease (AD) and ALS. They postulate how PGC1a could play a potential role in many neurodegenerative conditions [20].

Wenz, Rossi, and Rotundo et al. [21] have stated that elevated mitochondrial PGC1a may have beneficial effects on ageing. An elevated expression of PGC1a in muscle tissue during life has been shown to delay some conditions, such as muscle loss. These improvements were thought to be less decrease in mitochondrial function and a reduction of oxidative damage. Some have shown a possible protective role of PGC1a in Parkinson's disease while others have implicated PGC1a in Alzheimer's disease, Duchenne muscle dystrophy and ALS [22,23]. These studies suggest that PGC1a has the potential as a therapeutic agent in various neurodegenerative diseases.

\section{More Evidence for Mitochondrial Organelle Transfers as a Cell-Based Therapy}

There is compelling evidence that MOT may be a viable technology to treat other organ dysfunctions. Masuzawa, Black and Parak et al. [24] have shown in a magnificent study that transplantation of autologously derived mitochondria protects that heart from ischemia-reperfusion injury. Mitochondrial dysfunction and damage occurs during cardiac ischemia and affects cardiac function during reperfusion. Mitochondrial transplantation reduced cardiac damage and markedly improved cardiac ventricular function. In vivo and in-vitro studies showed 
mitochondria in the interstitial spaces, but were internalized by the cardiomyocytes 2-8 hours after transplantation. These results confirmed that transplanted mitochondria are easily internalized into cardiomyocytes within a few hours of a transfer, and they maintain viability and cardiac function by increasing ATP levels.

Recently Kitani, Kami et al. [25] have presented more compelling evidence that functionally isolated mitochondria transfer might be a promising therapy for various diseases. Their paper was entitled "Internalization of isolated functional mitochondria: involvement of macropinocytosis." They demonstrated that isolated mitochondria could be transferred into xenogeneic and homogeneous cells by simple co-incubation and that the method of cellular uptake involved macropinocytosis They proved the cellular uptake of the mitochondria by multiple sophisticated research techniques. The mitochondrial transfer improved the cellular viability in mitochondrial DNA-depleted cells and rescued mitochondrial respiratory function. These effects persisted for several days. Although problems exist and more needs to be done, these results are more evidence that MOT should be explored as a cellular-based therapy for serious diseases.

\section{Conclusion}

We have demonstrated in our research that isolated normal mitochondria can enter cancer cells and increase drug sensitivity and inhibit proliferation. This suggested that mitochondria could be powerful intracellular organelles forcell-based therapy.Further strong evidence has been presented in this commentary. This therapy has combined with other therapies and might improve or palliate patients with cancer and possibly neurodegenerative diseases. Organ transplantation has been done for years and now it is time to enter the era of cellular organelle transplantation. It has the potential to augment treatment of cancer, heart disease and possibly neurodegenerative disease.

We are beginning experiments with fibroblast mitochondria and neuronal cells. It is worth the effort to explore and perfect this technology. Especially if we can impact treatment of a disease like ALS. In order for MOT to be a viable technology, many obstacles must be overcome. We not only have to isolate normal mitochondria, but we also need to culture, expand and bank them for use when needed. We have cultured isolated normal mitochondria in our culture media (proprietary) for over 3 weeks; viability was documented by vital fluorescent stains. The cultured mitochondria also entered cancer cells and inhibited proliferation. The cultured mitochondria have been frozen for several weeks, thawed, and they still maintained viability. However, they stained less intense and the mitochondria population was less. We believe we are in the embryonic stage of MOT for cell-based therapy, but if we could enter the fetal stage and then birth, we possibly could impact the treatment of cancer and some neurodegenerative disorders. This is a difficult and complicated task, but if we reach our goal we might alleviate suffering and death from cancer and other dreaded diseases. The time for MOT is now!!

\section{References}

1. Warburg 0 , Wind F and Negleis E. On the Metabolism of Tumors in the Body. In: Warburg O. Ed, The Metabolism of Tumors, Constable, Princeton, 1930; 254-70.

2. Warburg 0. On the Origin of Cancer Cells. Science. 1956; 123(3191): 309-14.

3. Elliott RL, Jiang XP, Head JF. Mitochondria organelle transplantation: introduction of normal mitochondria into human cancer cells inhibits proliferation and increases drug sensitivity. Breast Cancer Res Treat. 2012; 136(2): 347-54. Doi: 10.1007/s10549-012-2283-2.

4. Nunnari J, Suomalainen A. Mitochondria in sickness and in health. Cell. 2012 16; 148(6): 1145-59. Doi: 10.1016/ j.cell. 2012.02.035.

5. Pasquier J, Guerrouahen BS, Al Thawadi H, Ghiabi P, Maleki M, AbuKaoud N, et al. Preferential transfer of mitochondria from endothelial to cancer cells through tunneling nano tubes modulates chemo resistance. J Transl Med. 2013; 11: 94. Doi: 10.1186/1479-5876-1194.

6. Kitani T, Kami D, Kawasaki T, Nakata M, Matoba S, Gojo S. Direct human mitochondrial transfer: a novel concept based on the endosymbiotic theory Transplant Proc. 2014; 46(4): 1233-6. Doi: 10.1016/j.transproceed.2013.11.133.

7. Menzies FM, Cookson MR, Taylor RW, Turnbull DM, ChrzanowskaLightowlers ZM, Dong L, et al. Mitochondrial dysfunction in a cell culture model of familial amyotrophic lateral sclerosis. Brain. 2002; 125: $1522-33$

8. Sathasivam S, Ince PG, Shaw PJ. Apoptosis in amyotrophic lateral sclerosis: a review of the evidence. Neuropathol Appl Neurobiol. 2001; 27(4): 257-74.

9. Shaw PJ, Eggett CJ. Molecular factors underlying selective vulnerability of motor neurons to neurodegeneration in amyotrophic lateral sclerosis. J Neurol. 2000; 247 Suppl 1: I17-27.

10. Bead MF. Mitochondrial dysfunction in neurodegenerative diseases. J Pharmacol Exp Ther. 2012; 342(3): 619-30. Doi: 10.1124/ jpet.112.192138.

11. Cortopassi GA, Wong A. Mitochondria in organism aging and degeneration. Biochim Biophys Acta. 1999; 1410(2): 183-93.

12.Zhang Y, Marcillat O, Giulivi C, Ernster L, Davies KJ. The oxidative inactivation of mitochondrial electron transport chain components and ATPase. J Biol Chem. 1990; 265(27): 16330-6.

13. Kong J, Xu Z. Massive mitochondrial degeneration in motor neurons triggers the onset of amyotrophic lateral sclerosis in mice expressing a mutant SOD1. J Neurosci. 1998; 18(9): 3241-50.

14. Youle RJ, van der Bliek AM. Mitochondrial fission fusion stress. Science. 2012; 337(6098): 1062-5. Doi: 10.1126/science.1219855.

15. Sheng ZH. Mitochondrial trafficking and anchoring in neurons: new insight and implication. J Cell Biol. 2014; 204(7): 1087-98. Doi: 10.1083/jcb.201312123.

16. Davis CH, Kim KY, Bushong EA, Mills EA, Boassa D, Shih T, et al. Transcellular degradation of axonal mitochondria. Proc Natl Acad Sci U S A. 2014; 111(26): 9633-8. Doi: 10.1073/pnas.1404651111.

17.Soubannier V, Rippstein P, Kaufman BA, Shoubridge EA, McBride HM. Reconstitution of mitochondria derived vesicle formation demonstrates selective enrichment of oxidized cargo. PLoS One. 2012; 7(12): e52830. Doi: 10.1371/journal.pone.0052830. 
18. Federico A, Cardaioli E, Da Pozzo P, Formichi P, Gallus GN, Radi E. Mitochondria, oxidative stress and neurodegeneration. J Neurol Sci. 2012; 322(1-2): 254-62. Doi: 10.1016/j.jns.2012.05.030.

19. Moreira PI, Zhu X, Wang X, Lee HG, Nunomura A, Petersen RB, et al. Mitochondria: a therapeutic target in neurodegeneration. Biochim Biophys Acta. 2010; 1802(1): 212-20. Doi: 10.1016/j. bbadis.2009.10.007.

20. Austin S, St-Pierre J. PGC1a and mitochondrial metabolism-emerging concepts and relevance in aging and neurodegenerative diseases. J Cell Sci. 2012; 125(Pt 21): 4963-71. Doi: 10.1242/jcs.113662.

21. Wenz T, Rossi SG, Rotundo RL, Spiegelman BM, Moraes CT. Increased muscle PGC-1alpha expression protects from sarcopenia and metabolic disease during aging. Proc Natl Acad Sci U S A. 2009; 106(48): 20405-10. Doi: 10.1073/pnas.0911570106.

22. Gong B, Chen F, Pan Y, Arrieta-Cruz I, Yoshida Y, Haroutunian V, et al. SCFFbx2-E3-ligase-mediated degradation of BACE1 attenuates Alzheimer's disease amyloidosis and improves synaptic function. Aging Cell. 2010; 9(6): 1018-31. Doi: 10.1111/j.1474-9726.2010.00632.x.

23. Qin W, Haroutunian V, Katsel P, Cardozo CP, Ho L, Buxbaum JD, et al. PGC-1alpha expression decreases in the Alzheimer disease brain as a function of dementia. Arch Neurol. 2009; 66(3): 352-61. Doi: 10.1001/ archneurol.2008.588.

24. Masuzawa A, Black KM, Pacak CA, Ericsson M, Barnett RJ, Drumm C, et al. Transplantation of autologously derived mitochondria protects the heart from ischemia-reperfusion injury. Am J Physiol Heart Circ Physiol. 2013; 304(7): H966-82. Doi: 10.1152/ajpheart.00883.2012.

25. Kitani T, Kami D, Matoba S, Gojo S. Internalization of isolated functional mitochondria: involvement of macropinocytosis. J Cell Mol Med. 2014; 18(8): 1694-703. Doi: 10.1111/jcmm.12316. 